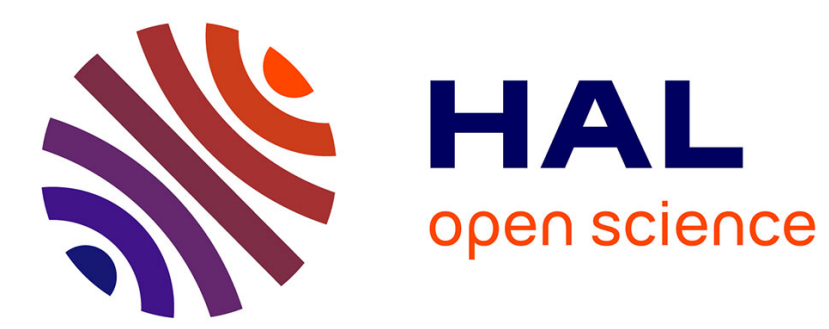

\title{
Désir de clinique
}

Olivier Ouvry

\section{To cite this version:}

Olivier Ouvry. Désir de clinique. Adolescence, 2008, Parano, 65 (3), 10.3917/ado.065.0627 . hal01444803

\section{HAL Id: hal-01444803 https://sorbonne-paris-nord.hal.science/hal-01444803}

Submitted on 24 Jan 2017

HAL is a multi-disciplinary open access archive for the deposit and dissemination of scientific research documents, whether they are published or not. The documents may come from teaching and research institutions in France or abroad, or from public or private research centers.
L'archive ouverte pluridisciplinaire HAL, est destinée au dépôt et à la diffusion de documents scientifiques de niveau recherche, publiés ou non, émanant des établissements d'enseignement et de recherche français ou étrangers, des laboratoires publics ou privés. 


\title{
Désir de clinique.
}

\author{
Olivier Ouvry, Maître de conférences \\ Laboratoire EA 3413, UTRPP, Université Paris 13
}

Novembre 2007

\section{Problématique.}

L'élaboration de ce numéro de la Revue a été l'occasion de discussions avec les membres du comité de pilotage ${ }^{1}$. Une des questions récurrentes fut de savoir s'il était pertinent de distinguer « parano $^{2}$ » et paranoïa à l'adolescence. Les discussions ont été fortes entre ceux qui considéraient ces deux termes comme à situer sur un même plan, et ceux qui insistaient sur la nécessité de les distinguer radicalement. La question ouverte était ainsi celle de la clinique. Elle constitue l'axe de cet article.

Ces discussions sont symptomatiques de la situation actuelle de cette dernière. Particulièrement confuse dans sa transmission ou son application, elle se trouve mise à mal dans les institutions de soin, dans les universités de médecine (psychiatrie) ou de psychologie. Il semble qu'un véritable flottement s'observe dans la définition de ce qui viendrait fonder les entités cliniques et organiser leur classification. La définition des supports sous-jacents (paradigmes) organisateurs de la clinique est devenue floue et inconsistante.

Or ces repères s'avèrent importants, d'autant que cet article s'intéresse au temps adolescent où l'expression symptomatique de la souffrance psychique est particulièrement trompeuse, redoublant les risques d'erreurs diagnostiques, lourdes de conséquences à cet âge de transition et de définition d'un projet de vie. Une prudence et une rigueur s'imposent ainsi.

Cette confusion s'inscrit dans une histoire. Elle a pour point de départ la diversité des « paradigmes » cliniques présents depuis quelques années. Nous en avons repéré deux nouveaux. Celui de la perspective dimensionnelle (Widlöcher D.,1990) et celui de la classification du DSM IV.

La perspective dimensionnelle trouve les principes de sa classification dans les effets cibles des divers médicaments à effets psychotropes. Ainsi, la dépression, par exemple, répond moins à une entité clinique à définir qu'à la réponse positive d'un ensemble symptomatique aux médicaments dits antidépresseurs. Le principe organisateur est ainsi celui pharmacologique. Il s'agit d'une « clinique » des effets des psychotropes. Ce sont eux qui sont le centre du principe classificateur. Pour le DSM IV, le paradigme est statistique. L'aspect symptomatique des manifestations du trouble vaut pour tout autre chose, puisque c'est de la fréquence de coprésences de certains d'entre eux que s'établit le diagnostic. Ainsi, une asthénie pour une pathologie autre que psychique (cancer par exemple) sera à ajouter à d'autres signes qui, par leur coprésence, établiront le diagnostic. La « réflexion » clinique a disparu' ${ }^{3}$.

\footnotetext{
${ }^{1}$ Tout particulièrement Marie-Christine Aubray, Olivier Douville, Odile Falque, Jean-Yves Le Fourn, Philippe Gutton, Patrice Huerre.

${ }^{2}$ Nous utilisons ce terme (qui n'a aucune occurrence dans la sémiologie ou la nosologie psychiatrique, dans la psychopathologie analytique) en référence aux interpellations usuelles des adolescents entre eux - supposant qu'il corresponde à un trait de personnalité consistant pour être si bien repéré par eux.

${ }^{3}$ Cela faisait dire à un psychiatre aussi important historiquement que Deniker qu'un concierge pouvait dorénavant faire un diagnostic psychiatrique...
} 
Dans les deux cas, nous avons le sentiment d'une abrasion de la dimension clinique. Le principe sous-jacent et ordonnateur se retrouve de fait sur le plan symptomatique, c'est-à-dire précisément celui que la clinique doit ordonner, et selon un référentiel extérieur au sujet, ce qui exclut la pathologie de son histoire ! Il y a là peut-être un effort de simplification, mais c'est au risque de la disparition... de la clinique ! C'est ce que nous observons aujourd'hui.

Ces deux nouveaux paradigmes sont venus se surajouter à celui antérieur, mis en place depuis le début du XXème siècle. Ce premier paradigme, historique, est celui de la nosologie kraepelinienne. Son repérage est, en revanche, plus complexe à opérer. Apparu en un temps où la psychiatrique tentait de se rapprocher de la médecine, il a reposé sur un effort de définition et de distinction d'entités nosologiques nécessairement abstraites, puisque ne s'étayant pas sur une étiologie connue (contrairement à la très grande majorité des affections somatiques). La notion de «maladies mentales » était donc une hypothèse. La démonstration de leur existence une gageure. C'est cela qui a été remis en cause dernièrement.

Artisanalement, néanmoins, Kraepelin a ordonné progressivement, par petites touches, les entités nosologiques, dans un effort de distinction les unes des autres (spécification/exclusion) et pour venir recouvrir, par leur adjonction, l'ensemble des pathologies mentales observables (contiguïté/complémentarité). Le tableau ainsi constitué s'est trouvé opérant et stabilisé. Il a fonctionné pendant quasiment un siècle.

Depuis sa remise en cause et sa perte de crédibilité, une validation de son entreprise est à tenter de façon à reporter sur le plan théorique la question de sa légitimité. Son élaboration oblige à un détour pour s'extraire de la confusion « politique ${ }^{4} »$ que nous venons de décrire. La singularité du temps adolescent peut-elle en consister un point de départ ? C'est l'hypothèse, apparemment paradoxale (l'adolescence risquant d'accuser cette confusion de par son processus même), que nous avançons.

\section{Le temps adolescent.}

Situé entre ceux de l'enfance et de l'adulte, il le temps du flottement, singulièrement sur le plan clinique où les aspects trompeurs observés ont déjà été évoqués. Ce flottement est tel qu'il conduit nécessairement à une exigence plus forte dans son approche et à la mise en place d'un système d'intelligibilité susceptible, tout au moins potentiellement, d'apporter quelques repères à cette clinique adolescente. L'approche psychopathologique, constituée à partir de la théorique analytique du pubertaire, s'impose ici en premier temps.

Prendre la voie théorique en préalable d'une approche clinique expose à une critique que l'on pourrait trouver dans des écrits de Freud. Il est possible, néanmoins, de la valider en arguant de la singularité du temps adolescent et de ses effets de brouillage, particulièrement opérant en clinique, et de l'exigence dans laquelle elle nous met d'une confrontation en second temps de ces acquis à notre question.

En s'inscrivant dans la continuité de Freud, il est possible de qualifier la nouveauté pubertaire comme l'apport du sexe non repéré dans l'infantile. Il s'inscrit dans les modifications morpho-physiologiques de la puberté des sujets des deux sexes, sans trouver d'écho dans le système phallique mis en place précédemment. C'est ainsi qu'il a été possible de

\footnotetext{
${ }^{4}$ Politique, car ce mouvement s'inscrit un ensemble plus large incluant celui de la mondialisation, de l'américanisation des mœurs, de la réduction du sujet à la personne, avec toute la valence adaptative qu'on attend de celle-ci dans un social qui a besoin d'elle comme force économique de consommation pacifiée. Cf. par ailleurs le mouvement «Sauvons la clinique » http://www.sauvons-la-clinique.org (SIUEERP).
} 
repérer cette nouveauté comme le « Féminin » (Ouvry O, 2001), présenté comme « réel pubertaire », c'est-à-dire comme une nouveauté échappant à toutes imaginarisation et symbolisation possibles.

Or venir repérer cette nouveauté pubertaire échappant au symbolique présuppose un acquis qu'il faut démontrer. Car comment envisager une théorisation susceptible de rendre compte de la «case vide », celle précisément mise au fondement de la nouveauté pubertaire (réel pubertaire) ? Capter le réel ne relève-t-il pas d'une gageure dès l'instant où les deux instances (réel et théorie) sont antinomiques?

Le réel est décrit comme l'impossible, l'au-delà du phallique, du symbolique. Or, il n'y a pas de formalisation théorique envisageable en dehors d'un discours, lui-même inscrit dans du symbolique.

Quant à l'imaginaire, il a horreur du vide, et vient recouvrir l'ensemble de ce sur quoi il s'applique - peut-être, précisément, afin d'en voiler le vide (réel) consubstantiel ! Nous retrouvons là une des caractéristiques décrite par Freud à propos du rêve et de sa production (Freud S., 1900), ou, plus avant, de l'hallucination chez le bébé venant de naître, lorsqu'elle vient en place de l'objet extérieur (Freud S., 1895). L'image est trompeuse, ou, plus précisément, l'imaginaire est trompeur - il vient habiller le désir inconscient refoulé de ses atours trompeurs, prompts à déjouer la vigilance de la censure et le principe de réalité. Il vient saturer la chose en prenant, dans tous les cas, l'apparence et les caractéristiques de ce qui provient des sens (de la sensorialité) pour illusionner le Moi et satisfaire à la nécessité de la réalisation du désir inconscient par les voies onirique et hallucinatoire. Il trompe la conscience en utilisant les images ${ }^{5}$ perceptives pour véhicule de ce qui leur est étranger dans leur principe les fantasmes et leur ambivalence, avec leurs effets de séduction, de chatoiement, voire de l'horreur ou de l'angoisse en cas d'échec du travail de l'imaginaire (cauchemars dans les rêves).

Lacan, plus tard, donnera une ampleur plus radicale à cette perspective en articulant l'origine du Moi à l'instance imaginaire à partir du reflet de l'image du corps dans le miroir (Lacan J. 1936). Ainsi tout ce qui est produit par cette instance - le Moi, la conscience devient suspect, par le fait du caractère d'illusion et de tromperie, de confusion et de doute de ce qui l'origine, à savoir la voie obligée de l'imaginaire.

L'imaginaire, porteur en soit d'un principe de tromperie, se retrouve donc au fondement de ce qui donne pourtant aux notions d'existence et de maîtrise un semblant de consistance (l'instance du Moi). Or toute construction théorique en est une émanation. Peut-il être, dans ces circonstances, envisageable de trouver un cadre théorique susceptible de déjouer l'instance de l'imaginaire, avec ses fantasmes de maîtrise et de "scientisme », et de spécifier du réel qui, par nature, lui échappe ? Quelle serait la construction méthodologique qui pourrait contenir les effets d'illusions et de satisfactions imaginaires et assignerait au travail le chercheur sur des objets obéissant à des règles qu'il n'aurait pas fondées ?

Au réel, qui est l'impossible, et à l'imaginaire, suspect, doit donc « s'opposer » une théorisation singulière. Elle aurait pour caractéristique de pouvoir capter le réel, par une voie nécessairement symbolisée, et de déjouer les pouvoirs falsificateurs de l'imaginaire et de maîtrise du moi. Qui plus est, elle devra se formaliser dans une méthodologie qui permette une approche nouvelle du problème, ouvrant à d'autres perspectives. C'est celle que l'on peut situer à l'orée du XXème siècle dans le structuralisme.

\footnotetext{
${ }^{5}$ Ce qui nous permet de différencier image et imaginaire, en tant que fonction venant s'exercer sur les images pour ce dernier.
} 


\section{La voie structuraliste.}

Fondée à partir de la linguistique (Ferdinand de Saussure, 1916), l'approche structuraliste a essaimé dans l'ensemble des Sciences Humaines offrant un véritable renouveau de l'abord des faits humains. Que ce soit en anthropologie (Claude Lévi-Strauss), en littérature (Roland Barthes), en philosophie (Maurice Merleau-Ponty) ou en psychanalyse (Jacques Lacan ${ }^{6}$ ) (Dosse F ., 1992), son influence a été majeure, au point de pouvoir qualifier le XXème siècle et son histoire autour de son avènement et de ses suites.

Offrir une radicalité dans la définition de ce qui est observé en Sciences Humaines, en termes de signe ; détaché d'un référent (réalité) ; rattaché à une image issue de la sensorialité (image acoustique, appelée signifiant par F. de Saussure) et un concept (appelé signifié) ; séparés l'un de l'autre par l'arbitraire inscrit sous forme de barre $(\mathrm{s} / \mathrm{S})$; inséré et différencié par coupure de l'ensemble des autres signes présents définissant sa « valeur »; indépendamment de toute historisation des mots ou concepts (prévalence de la synchronie, rejet de la diachronie, de l'histoire et de la métaphysique) ; articulé aux autres signifiants selon des règles de la spatialisation (topologie), venait en effet changer radicalement la donne du fait humain. Une structure travaille sur des objets concrets, telles les « images acoustiques », sensorialisées (signifiants), et vient les articuler les uns aux autres selon une topologie qui intègre le principe d'une modification de la configuration de l'ensemble de ses éléments par l'ablation d'un de ceux-ci. Une structure donnée devient ainsi porteuse de ce qui la constitue, en elle-même, en tant qu'éléments présents, mais également en tant qu'éléments absents (case vide ${ }^{7}$ ), son équilibre portant la trace de ce qui l'origine ${ }^{8}$.

Il y a ainsi désolidarisation entre un niveau de la réalité, non pris en compte (référent), un niveau imaginaire, intégrant le concept et ses valences historique et métaphysique (sens), annulé par la nature arbitraire de son lien avec ce qui devient la substance définie, et unique, de l'étude structurale : le signifiant. L'ensemble des jeux complexes par lesquels il se trouve articulé aux autres signifiants qui l'entourent obéissent à des lois, celles du langage. Se distinguent ainsi, peut-on observer, une réalité, un imaginaire et un symbolique, genèse de ce qui deviendra $\mathrm{RSI}^{9}$ dans la théorisation lacanienne.

Ce projet d'éradication de l'imaginaire interne - souvenirs, sens - et externe - réalité, histoire - évoque le projet analytique, tel que Freud l'a initié. C'est, de fait, dans cette perspective que le rêve devient un rébus, chaque image de celui-ci étant rapportée, par le jeu des associations libres, à autant d'éléments qui, articulés les uns aux autres sous forme de phrase, forment une structure plus ou moins cohérente, à compléter, porteur de la réalisation d'un désir inconscient refoulé. L'interprétation, en tant qu'élément rajouté pour redonner une cohérence à cet ensemble, y trouve sa définition : d'épingler, par déduction logique, structurale, les exclusions que suppose une telle configuration - c'est-à-dire les signifiants dont l'exclusion (refoulement) est nécessaire à cette constitution donnée, à sa cohérence, en tant que phrase. C'est de cette déduction logique ${ }^{10}$ qu'elle trouve la justification de son introduction possible

\footnotetext{
${ }^{6}$ Lacan se différenciera de ces autres auteurs en apportant une nuance de taille ; celle de la décomplétude de l'Autre.

${ }^{7}$ Ce que Freud repérait, dans sa théorie du rêve, comme « l'ombilic du rêve. »

${ }^{8}$ «En premier lieu, une structure offre un caractère de système. Elle consiste en éléments tels qu'une modification quelconque de l'un d'entre eux entraîne une modification de tous les autres. En second lieu, tout modèle appartient à un groupe de transformations dont chacune correspond à un modèle de la même famille, si bien que l'ensemble de ces transformations constitue un groupe de modèle.

Troisièmement, les propriétés indiquées ci-dessus permettent de prévoir de quelle façon réagira le modèle, en cas de modification de l'un de ses éléments. Enfin, le modèle doit être construit de telle façon que son fonctionnement puisse rendre compte de tous les faits observés. » (Lévi-Strauss Cl., 1958, p. 306)

${ }^{9}$ RSI, soit Réel, Symbolique et Imaginaire, et leurs articulations borroméennes.

${ }^{10}$ Cf. Freud S. (1933a, p. 20) : « Nous intervenons alors de notre propre chef, complétons les allusions,
} 
aux dires du patient. Il n'y a pas d'autre fondement théorique et d'autre éthique possibles à l'interprétation, dès l'instant où il s'agit de garantir qu'elle ne soit pas porteuse des signifiants propres au psychanalyste - voire aux excursions de ses fantasmes dans ceux de son patient.

Il est cohérent ainsi de présenter Freud comme préstructuraliste (structuraliste à son insu), dans la continuité de ce que Lacan avance dans L'instance de la lettre ou la raison depuis Freud $^{I l}$ (Lacan J., 1957) - car seule cette approche pouvait permettre la révélation de ce qui était présent en deçà de la représentation, l'inconscient en premier lieu. C'est également ce qui nous a fait penser que Freud était, à son insu, un théoricien du pubertaire (Ouvry O., 2004) car il esquissait, par déduction logique, des voies de théorisation possible du pubertaire, non développées par inexistence de moyens de formalisation disponibles à son époque. Ces voies ont trouvé leurs développements dans des apports issus d'une approche structuraliste, par exemple dans le déplacement de ce qu'il décrivait sous l'appellation énigmatique d'intégration du vagin dans la génitalité à partir de la puberté (et pas avant), ou, moins directement, d'une libido exclusivement rapportée au masculin - en tant que correspondant à ce qui a pu être repéré sous les apories lacaniennes de : «il n'y pas de signifiant pour le sexe de la femme », et, par déductions logiques, « La femme n'existe pas » et « il n'y a pas de rapport sexuel ».

\section{Processus adolescent et structuralisme.}

Il peut être tentant, à ce niveau du raisonnement, de suggérer que si le réel pubertaire peut être repéré par une approche structuraliste, alors le sujet qui en est l'objet s'y trouve lui-même introduit, par essence. Ainsi, l'idée de l'avènement de la nouveauté pubertaire accompagnant, chez ce nouvel adolescent, un développement de la perspective structuraliste comme nouvelle donne psychique - venant qualifier ce qui serait nouveau sur le plan de sa cognition ${ }^{12}-$ se déduirait volontiers.

Cette idée peut trouver un autre support dans une coïncidence relevée par une autre voie : celle historique. Il est en effet remarquable que l'apparition du structuralisme, sur le plan de l'histoire des idées, soit contemporaine de celle de l'adolescence en tant que nouvelle donne sociologique.

Le structuralisme s'impose progressivement dans les différentes Sciences Humaines, comme il a déjà été précisé, tout le long du XXème siècle à partir de son point d'origine linguistique du début de ce siècle. Mais ses prémisses se retrouvent bien avant cela, déjà chez

\footnotetext{
tirons des conclusions irréfutables, formulons ce que le patient n'a fait qu'effleurer. Ceci pourrait donner l'impression que nous laissons notre esprit et notre bon plaisir jouer avec le matériel que le rêveur met à notre disposition et que nous en abusons pour mettre par interprétation dans ses propos ce qui ne s'aurait s’y interpréter »... Et Freud nous renvoie alors à la littérature sur ce sujet, et à l'application de cette technique sur nous-mêmes «pour vous convaincre de la manière contraignante dont se déroule un tel travail d'interprétation ».

${ }^{11}$ La citation exacte de Lacan à propos de ce point est la suivante : « Dès l'origine on a méconnu le rôle constituant du signifiant dans le statut que Freud fixait à l'inconscient d'emblée et sous les modes formels les plus précis. Ceci pour une double raison, dont la moins aperçue naturellement est que cette formalisation ne suffisait pas à elle seule à faire reconnaître l'instance du signifiant, car elle était, à la parution de la Traumdeutung, très en avance sur les formalisations de la linguistique auxquelles on pourrait sans doute démontrer qu'elle a, par son seul pesant de vérité, frayé la voie. » (Lacan J., 1957, pp. 512-513) Ce texte est celui dans lequel Lacan fait le lien entre les deux axes du travail du rêve, tels que dégagés par Freud (condensation et déplacement), les deux axes des opérations du langage, tels que dégagés par F. de Saussure (combinaison, sélection), et l'analogie que propose Roman Jakobson dans son étude sur les aphasies avec la métaphore et la métonymie. Cet article passe par ces mêmes associations.

${ }^{12}$ Il serait intéressant de confronter cette idée avec la notion de pensée formelle, décrite par Piaget comme nouvelle à la puberté.
} 
Freud, comme Lacan nous le souligne, mais également le long du XIXème siècle (positivisme d'Auguste Comte, notion du transcendantal chez Kant, Marx...). Or c'est bien à cette même période que se dévoile progressivement l'adolescence comme force sociale nouvelle.

Il a déjà été développé dans cette Revue (Ouvry O. \& coll, 1996) l'idée que l'adolescence, en tant qu'entité sociologique, apparaît sous un mode balbutiant à la Révolution Française $^{13}$, que son développement ne se fera que progressivement tout au long du XIXème siècle, se manifestant socialement au début du XXème (bande des Apaches, approche psychopathologique telle que défendue dans les «patronages » mis en place par Rollet) et trouvant son apogée lors de l'ordonnance de 1945. Or c'est sur cette même période qu'apparaît le structuralisme, en tant que projet nouveau dans l'abord des faits humains ${ }^{14}$.

Il y aurait ainsi une histoire commune de l'adolescence, sur un plan individuel ou social, et du structuralisme. Peut-on, alors, retourner l'apparente coïncidence historique de leur apparition en saisi d'un mouvement commun à l'expression différenciée selon les plans où elle s'observe, les rendant consubstantiels l'un à l'autre en tant qu'acquisitions ontologique ( « cognitive » et psychologique - processus adolescent), sociale (conscience collective - entité sociologique de l'adolescence) et épistémologique (histoire des concepts - structuralisme) conjointes $^{15}$ ? L'adolescence y trouverait une nouvelle approche dans sa définition.

\section{La nosologie kraepelinienne.}

Mais, et en poursuivant la veine de la coïncidence historique, de façon contemporaine à ces précédents apports se trouve également l'apparition de la nosologie psychiatrique. Nous entendons par celle-ci la mise en place de la clinique psychiatrique classique, rapporté à la démarche de Kraepelin principalement ${ }^{16}$. C'est lui qui nous oblige à la rigueur nosologique, et à la différenciation des tableaux cliniques au point de les considérer comme s'excluant les uns aux autres et recouvrant, par nécessité, l'ensemble des entités cliniques observés - démarche particulièrement remarquable pour instituer un cadre nosologique des affections psychiatriques en l'absence d'étiologies dans les affections psychiques.

Dans cette perspective, un signe sémiologique doit faire l'objet d'une « lecture » pour être rapporté à une entité nosologique, et à une seule, à laquelle il n'est pas spécifique par nature. Il n'a, en effet, de pertinence en soi que par le fait qu'il se trouve inclus dans un

\footnotetext{
${ }^{13}$ L'idée de l'avènement de l'adolescence à la Révolution Française peut surprendre, sinon par une réflexion que Freud a lui-même initié. L'idée que la volonté d'un seul devienne la Loi de tous est à l'origine de son ouvrage Totem et tabou (Freud S., 1915) (meurtre du père de la horde primitive). Elle se retrouve dans la mise en place de la première religion monothéiste (mise à mort de Moïse - cf. Le Moïse et le monothéisme (Freud S., 1939)). Ce principe, appliqué à la mise à mort du Roi, peut apporter quelques éclaircissements à notre question. La transmission de son patrimoine à sa descendance était en effet l'objet de la royauté et des nobles, seulement. La mise à mort de celui qui les incarnait transforme cette question de quelques uns en règle pour tous, et étend la question de la transmission aux générations suivantes à toutes les classes de la société. Or qui dit transmission, dit problème de celle-ci, et spécification de ceux qui en sont porteurs. L'entité sociologique des adolescents en émane potentiellement.

${ }^{14}$ La thèse de Claude Lévi-Strauss, premier texte fondateur de l'approche structuraliste dans les Sciences Humaines, est soutenue à Paris en 1947, et éditée en 1949 (Lévi-Strauss C., 1949).

${ }^{15}$ Nous faisons ici une analyse historique structurale, opérant une bascule entre un plan manifeste, descriptif et inerte, à celui structural où un signifiant apparaît historiquement, en lien avec un signifié aux multiples significations, déclinées sur autant de strates qu'il y en a entre l'individuel et le collectif. ${ }^{16}$ Nous ajoutons « principalement », car il n'est pas le seul à y avoir contribué (Freud entre autres, y a participé). C'est néanmoins Kraepelin qui a promulgué cette démarche, en se donnant pour objectif une classification cohérente et totalisante de l'ensemble des pathologies psychiatriques - démarches centrée sur l'articulation des tableaux dans leurs dimensions sémiologique (synchronique) et évolutive (diachronique).
} 
ensemble d'autres, cohérents entre eux quant à leur « valeur» (pour reprendre un terme de F. de Saussure), et venant alors lui donner sa correspondance en terme nosologique ${ }^{17}$. Sans cela, il n'y a pas de renvoi sur le plan diagnostic (nosologique).

Nous retrouvons ici le sujet de notre article (la parano et la paranoïa à l'adolescence), mais également ce qui en a ordonné jusqu'alors son contenu, à savoir l'approche structurale. Car, de fait, le projet kraepelinien, de s'affranchir de la dimension de l'apparence des affections psychiques pour en retrouver le support constitutif, seul différenciateur au sein de la pluralité de leurs présentations, prend, à son insu, tout comme Freud, le courant structural comme principe organisateur. On retrouve également le souhait de s'affranchir de la dimension imaginaire, c'est-à-dire de l'expression manifeste de la pathologie, brouilleur des tableaux cliniques présents, pour atteindre celui de leur consistance en terme de structure (cf. les trois catégories principielles : Névrose, Psychose et Perversion, et leurs déclinaisons en entités nosologiques).

C'est dans cette perspective qu'il devient possible de poser une différenciation radicale de la parano et la paranoïa, à l'adolescence notamment. La première relèverait de la dimension imaginaire, c'est-à-dire des manifestations (sémiologie) des troubles psychiques, la deuxième de celle structurale (nosologie), organisatrice de ceux-ci. Ce point, important à nos yeux, apporte un repérage précis pour distinguer deux niveaux d'interprétation clinique, et deux notions qui, autrement, resteraient floues, incertaines et d'usage approximatif en clinique.

\section{La « parano » adolescente.}

De paranoïa en termes d'entité nosologique à l'adolescence, il ne nous a pas été donné d'en observer - ce qui n'exclue pas qu'elle puisse être présente, au moins en termes de structure. Il peut sembler que cette pathologie ait besoin de temps pour se déployer et développer, que l'adolescent n'a pas encore trouvé, vu son jeune âge (on parle de psychose « chronique »). En revanche, de parano, en tant que modalité expressive d'une affection encore à qualifier, nous en avons vu, et même souvent à l'adolescence. Est-ce un trait particulier de ce temps-là ?

L'effroi pubertaire, rapporté pour nous à celle de la nouveauté pubertaire, c'est-à-dire de la case vide que nous repérions précédemment en tant que réel pubertaire, semble de fait, par sa radicale nouveauté à cet âge, susciter un mouvement phobique général, un appel du vide (un vertige), chez l'adolescent - vide que peut venir voiler tout élément disponible à la panoplie de l'adolescent, que ce soit son corps propre, l'objet quel qu'il soit, avec sa tonalité addictive, ou, plus simplement, l'autre, et l'autre de l'Autre sexe notamment. C'est probablement, également, cette singularité du temps adolescent d'affleurement de la case vide qui donne, à ceux qui le traversent, ce côté si subversif auprès des générations qui les ont précédées. Avant qu'ils ne la recouvrent et en arrivent au temps de la pacification de cette nouveauté pubertaire, de voilement de ce réel pubertaire, ils s'affrontent à cet effroi si créateur de nouveaux équilibres qui interrogent ceux jusqu'alors mis en place ${ }^{18}$ - jusqu'à faire s'interroger sur la « parano »

\footnotetext{
17 « La définition du contenu d'un phonème dépend de la place qu'il occupe dans le systèmes des phonèmes dont il s'agit (...). Un phonème ne possède un contenu phonologique définissable que parce que le système des oppositions phonologiques présente une structure, un ordre déterminé. » (Troubetzkoy, 1964, p.69) «D'où l'on peut dire que c'est dans la chaîne du signifiant que le sens insiste, mais qu'aucun des éléments de la chaîne ne consiste dans la signification dont il est capable au moment même. » (Lacan J., 1957, p. 502)

18 « Je me dois à la société, c'est juste, - et j'ai raison. - Vous aussi vous avez raison, pour aujourd'hui. Au fond, vous ne voyez en votre principe que poésie subjective : votre obstination à regagner le râtelier universitaire, - pardon ! - le prouve ! Mais vous finirez toujours comme un satisfait qui n'a rien fait, n'ayant rien voulu faire. Sans compter que votre poésie subjective sera toujours horriblement fadasse. Un jour, j'espère, - bien d'autres espèrent la même chose, - je verrai dans votre principe la poésie objective, je la verrai plus sincèrement que vous le feriez ! - Je serai un travailleur : c'est l'idée qui me
} 
adolescente les adultes qui les entourent.

Dans ce ressort phobique se trouve certainement celui de la parano adolescente. La projection de cet effroi sur l'autre, qu'il soit autre de la réalité, ou autre plus ou moins intériorisé (le corps par exemple), est à l'origine de ces expressions à ne pas confondre avec ce qui viendrait témoigner d'une structuration (sous ce mode) engageant la pathologie.

\section{Perspectives.}

Il est certainement singulier de retrouver le structuralisme associé à ce qui peut lui être si dissemblable en premier abord : le processus adolescent, la nosologie kraepelinienne, la théorisation analytique. Mais n'est-ce pas en revenant à leur mouvement commun (tentative d'éradication des effets de l'imaginaire, référence à la structure en tant que langage) que leurs principes peuvent le mieux se saisir, offrant des voies de passage de l'un à l'autre, heuristiques quant à la question de la clinique ?

Une des critiques les plus acérées du structuralisme a été, en le confinant à ce qui est sa méthode, de le considérer comme réducteur des problèmes de l'homme, les ramenant à des configurations mathématiques abstraites et désubjectivantes. Mais l'hypothèse du structuralisme n'est-elle pas, justement, là ? À savoir de mettre en place une méthodologie de défrichage et de déblayage de ses scories imaginaires le terrain d'observation pour mieux y observer les manifestations du sujet et l'écoute de ses expressions dans quelques domaines que ce soi (anthropologie, sociologie, philosophie, psychanalyse, littérature...) ? N'est-ce pas dans la mise en place de conditions méthodologiques d'une observation possible que les garanties de la prise en compte du sujet sont ainsi les mieux assurées (le principe de la règle des associations libres en psychanalyse est là), le but n'étant pas de l'enfermer dans une idéologie a priori ?

Ainsi, le paradigme théorique de la nosologie kraepelinienne, qui se trouvait en projet de cet article, se présente comme structuraliste. Il repose sur l'effort de se défaire de l'aspect purement manifeste des pathologies mentales pour accéder à ce qui les ordonne et les différencie. La structure du langage en est la base, le sujet sa conséquence.

Cet article, intitulé « Désir de clinique », intervient à un moment où elle se trouve particulièrement mise à mal dans tous ses champs d'application. Sa validation devient une nécessité. L'hypothèse à laquelle nous sommes conduits est qu'une approche clinique respectueuse du sujet est, par essence, structuraliste - même à l'insu des praticiens qui l'exercent. Elle est à situer sur le plan du désir, car le sujet s'y trouve impliqué, jusqu'à celui qui en porte la réflexion.

retient, quand les colères folles me poussent vers la bataille de Paris - où tant de travailleurs meurent pourtant encore tandis que je vous écris ! Travailler maintenant, jamais, jamais : je suis en grève. » (Rimbaud, 1871) 


\section{Résumé.}

Notre propos est, à travers une tentative de clarification des termes de parano et paranoïa à l'adolescence, de montrer l'articulation possible d'une approche logique, introduite par l'approche structurale, avec le mouvement même du processus adolescent. Cela se centre sur la notion de «case vide », telle qu'elle peut se retrouver sur les notions de Féminin et de réel pubertaire.

Cela conduira à reprendre les principes théoriques du structuralisme, la théorisation analytique du pubertaire et la nosologie psychiatrique, avec pour objectif, au-delà de sa complexité apparente, d'articuler un ensemble de données pour les rendre co-intelligibles, où une clinique peut se trouver validée, spécifiquement adolescente.

\section{Bibliographie.}

Dosse François, Histoire du structuralisme, Tome 1, Ed La découverte, Paris, 1992.

Ferdinand de Saussure, 1916, Cours de linguistique générale, Payot, Paris 1986 (1972). Freud S., 1895, Esquisses d'une psychologie scientifique, in Naissance de la psychanalyse, PUF, Paris, 1956.

Freud S. (1900), L'interprétation des rêves, Gallimard, Paris, 1925.

Freud S. (1912), Totem et Tabou, Payot, Paris 1947.

Freud S. (1933a), Nouvelles conférences d'introduction à la psychanalyse, XXIXe conférence : révision de la théorie du rêve, Gallimard, coll. Folio essais, Paris, 1984. Freud S. (1939), Moïse et le monothéisme, Gallimard, Paris, 1948.

Lacan J. 1936 Le stade du miroir comme fondateur de la fonction du Je, in Ecrits, Ed. du Seuil, Paris 1966 (pp. 93-100).

Lacan J., 1957, L'instance de la lettre dans l'inconscient ou la raison depuis Freud, in Ecrits, Ed. du Seuil, Paris 1966 (pp. 493-529).

Lévi-Strauss Cl., Les structures élémentaires de la parenté, Paris, La Haye, Mouton et Co., 1967.

Lévi-Strauss Cl., 1958, Anthropologie structurale, Plon.

Ouvry O. Marty F. Bourquin J. (1996). Évolution de la notion de travail associée à celle d'adolescence au cours du XIXe et au début du XXe siècle. Adolescence $1996 N^{\circ} 28.81-91$. Ouvry O. (2001). Le Féminin comme nouveauté pubertaire. Le Féminin : un concept adolescent ? sous la direction de Serge Lesourd, Ed Erès, coll. Le Bachelier. 49-76. Ouvry O. (2004). Freud : théoricien du pubertaire ?. Clinique Méditerranéenne, $N^{\circ} 70,241$ 252.

Rimbaud A., 1871, Lettre dite du « Voyant » à G. Izambard, 13 mai 1871.

Troubetzkoy, 1964, Principes de phonologie, trad. Cantineau J., Klincksieck, Paris. Widlöcher D., Les psychotropes, une manière de penser le psychisme ?, Les empêcheurs de tourner en rond, Paris, 1990. 